Relations industrielles

Industrial Relations

\title{
The Canadian Labour Market, by Arthur Kruger and Noah M. Meltz, Editors, Center for Industrial Relations, University of Toronto, 1968, 312 pages.
}

\section{Jean Boivin}

Volume 24, numéro 1, 1969

URI : https://id.erudit.org/iderudit/028002ar

DOI : https://doi.org/10.7202/028002ar

Aller au sommaire du numéro

Éditeur(s)

Département des relations industrielles de l'Université Laval

ISSN

0034-379X (imprimé)

1703-8138 (numérique)

Découvrir la revue

Citer ce compte rendu

Boivin, J. (1969). Compte rendu de [The Canadian Labour Market, by Arthur Kruger and Noah M. Meltz, Editors, Center for Industrial Relations, University of Toronto, 1968, 312 pages.] Relations industrielles / Industrial Relations, 24(1), 221-222. https://doi.org/10.7202/028002ar

Tous droits réservés (C Département des relations industrielles de l'Université Laval, 1969
Ce document est protégé par la loi sur le droit d'auteur. L’utilisation des services d’Érudit (y compris la reproduction) est assujettie à sa politique d'utilisation que vous pouvez consulter en ligne.

https://apropos.erudit.org/fr/usagers/politique-dutilisation/ 
problem of public finance (Part 3) in dealing with taxation, public expenditures, social security, the fiscal policy and the public debt.

It is only in the final part (Foreign trade) that the author goes on the international scene by examining the Canadian foreign trade and the tariff policy and the problem of the balanced of payments.

Completed by a large bibliography and a good number of statistical tables, this book is a must for all those interested as well in economics as in labor relations in Canada.

\section{Jean SEXTON}

The Canadian Labour Market, by Arthur Kruger and Noah M. Meltz, Editors, Center for Industrial Relations, University of Toronto, 1968,312 pages.

Ce livre contient les résultats de recherches conduites par un groupe d'économistes à l'Université de Toronto sur des problèmes de main-d'oeuvre.

Le premier article d'Arthur Kruger, examine l'allocation des ressources humaines par les forces du marché et ce, de deux façons. D'abord, d'un point de vue théorique idéal; ensuite, d'après le degré d'éloignement du marché du travail de cet idéal théorique.

Le professeur Kruger discute également des politiques de main-d'oeuvre à la lumière de la théorie économique.

Il termine en mentionnant que les coûts et les avantages des programmes de main-d'oeuvre doivent être calculés d'après ce qu'ils représentent à la fois pour l'individu et pour la société.

Le deuxième article, celui de Samuel Hollander, présente le développement de la pensée en économique du XIXe siècle au sujet du fonctionnement du marché du travail.

Il montre que plusieurs questions économiques d'actualité étaient également discutées par les économistes des siècles passés. Ainsi, il présente les vues d'Adam Smith et de J. S. Mill sur l'influence du gouvernement et des syndicats sur la mobilité (ou l'immobilité) des travailleurs.
Hollander termine son article en soulignant que, pour les classiques, le chômage ne pouvait être que temporaire à cause de la flexibilité à la baisse des prix et des salaires monétaires.

Dans le troisième article, Yehuda Kotowitz montre comment les changements technologiques ont pour effet d'entraîner un déplacement des travailleurs. Il utilise un cadre théorique pour comparer la relation qui peut exister entre le progrès technique et le chômage. Par la suite, il présente des études empiriques sur l'expérience canadienne à ce sujet avant et après la deuxième guerre mondiale.

En terminant, le professeur Kotowitz montre que l'impact du progrès technique sur l'emploi est tout à fait différent selon que l'on considère l'économie globale ou que l'on considère les entreprises ou les industries. Le progrès technologique déplace les individus dit-il mais il permet simultanément la croissance de l'emploi et des salaires en général.

Dans son article, John Winder met en lumière une des questions les plus controversées parmi les économistes actuellement, à savoir si les hauts taux de chômage qui ont existé entre 1957 et 1963 étaient le résultat d'une demande globale déficiente ou de changements structurels dans l'économie.

Le professeur Winder définit le chômage structurel et montre comment il diffère des autres formes de chômage. Il classifie les différents types de chômage, cyclique, saisonnier, technologique, structurel et frictionnel, d'après leurs causes et d'après les politiques appropriées pour les combattre.

Selon lui, la lutte contre le chômage implique un dosage adéquat entre deux genres de politiques: les politiques traditionnelles fiscales et monétaires et les politiques de main-d'oeuvre. La question étant de savoir combien de chacune doit être utilisée.

Le chapitre suivant est un apport formidable à la recherche sur la maind'oeuvre en général. Il s'agit d'un recueil de toute l'information actuellement publiée au Canada sur les problèmes de main-d'oeuvre.

En plus d'indiquer le contenu des diverses publications, l'auteur en fait la critique et souligne ce qu'il manque 
comme information indispensable sur le marché du travail.

Finalement, l'article se termine par une série de recommandations, notamment, la nécessité de se tenir au courant des développements du marché du travail au moins annuellement comme on le fait aux Etats-Unis dans le "Manpower Report of the President". De plus, il souligne que des statistiques additionnelles devraient être fournies sur l'emploi et les salaires par occupation.

Dans les deux derniers articles Ian Drummond et David Stager examinent les relations entre le système éducationnel et les marchés du travail.

Pour le professeur Drummond les investissements dans l'éducation n'ont de sens que si leurs bénéfices sociaux dépassent leurs coûts sociaux. C'est pourquoi il présente différentes façons d'évaluer le rapport coûts/bénéfices et termine en proposant que l'analyse de ce rapport par les politiciens soit effectuée en conjonction avec les prévisions de l'évolution de la main-d'oeuvre.

David Stager, pour sa part, discute des difficultés conceptuelles qui existent lorsqu'on veut mesurer la « production 》 des institutions éducationnelles post secondaires. Cependant, il ne s'occupe que de l'aspect «instruction》 laissant de côté la «production》 de la recherche.

En terminant, il faut mentionner que ce livre est l'une des publications les plus intéressantes qui traitent des problèmes de main-d'oeuvre canadiens actuels.

\section{Jean BOIVIN}

Ressources humaines pour le développement industriel, Bureau international du travail, Genève, 1967, 276 pages.

Cette publication du BIT rassemble des documents préparés par le BIT en vue d'un colloque international de la Nouvelle organisation des Nations Unies pour le développement industriel (ONUDI).

Ces documents se répartissent en deux séries. Les documents de la première série, intitulée «Le problème de la maind'oeuvre » traitent de la formation professionnelle et de l'utilisation de la maind'oeuvre en vue du développement industriel.
La seconde série de documents intitulée \& autres questions exigeant une politique générale 》 porte «sur la participation sociale au développement industriel met l'accent sur l'importance d'une collaboration entre les différents groupes sociaux, tout particulièrement les employeurs, les travailleurs et leurs organisations, et de leur participation active au processus d'industrialisation, puis passe en revue les fins auxquelles cette participation serait utile et les formes qu'elle peut revêtir ».

Une première étude intitulée «Besoins en main-d'oeuvre qualifiée $\gg$ se conclut sur une série de recommandations pertinentes mettant l'accent sur les méthodes à employer afin de prévoir avec le maximum de précision l'évolution des besoins en main-d'oeuvre considérée non seulement sous l'angle quantitatif, mais également sous l'angle qualitatif (niveau de qualification professionnelle).

Une seconde étude consacrée aux programmes d'éducation et de formation préconise l'intégration sur la base de leur complémentarité de l'enseignement général et de l'enseignement technique et professionnel et tend à dégager les grandes lignes d'une méthodologie qui permettrait de dégager les objectifs généraux en matière d'éducation et de formation puis de les détailler ensuite en fonction des divers secteurs d'enseignement.

Cette étude comporte également diverses suggestions concernant l'organisation et le financement des divers programmes. Elle se termine sur quelques propositions précises de l'action internationale dans ce domaine.

Les deux dernières études de la première partie sont consacrées respectivement à « l'utilisation effective de la main-d'oeuvre en vue de l'industrialisation 》 et à la «formation professionnelle dans les petites entreprises de transformation des pays en voie de développement ».

La seconde partie contient une intéressante étude intitulée \&la participation sociale au développement industriel $\gg$.

«La mise sur pied d'une organisation satisfaisante des rapports humains et sociaux dans le secteur industriel et en particulier au sein des entreprises industrielles » est considérée comme une condition favorable au développement. 